\title{
ORMDL3 regulates poly I:C induced inflammatory responses in airway epithelial cells
}

\author{
Gemma Laura', Yi Liu', Kieran Fernandes', Saffron A. G. Willis-Owen', Kazuhiro Ito ${ }^{1,2}$, William O. Cookson', \\ Miriam F. Moffatt ${ }^{1}$ and Youming Zhang ${ }^{1 *}$
}

\begin{abstract}
Background: Oroscomucoid 3 (ORMDL3) has been linked to susceptibility of childhood asthma and respiratory viral infection. Polyinosinic-polycytidylic acid (poly I:C) is a synthetic analog of viral double-stranded RNA, a toll-like receptor 3 (TLR3) ligand and mimic of viral infection.

Methods: To investigate the functional role of ORMDL3 in the poly l:C-induced inflammatory response in airway epithelial cells, ORMDL3 knockdown and over-expression models were established in human A549 epithelial cells and primary normal human bronchial epithelial (NHBE) cells. The cells were stimulated with poly l:C or the Th17 cytokine IL-17A. IL-6 and IL-8 levels in supernatants, mRNA levels of genes in the TLR3 pathway and inflammatory response from cell pellets were measured. ORMDL3 knockdown models in A549 and BEAS-2B epithelial cells were then infected with live human rhinovirus (HRV16) followed by IL-6 and IL-8 measurement.

Results: ORMDL3 knockdown and over-expression had little influence on the transcript levels of TLR3 in airway epithelial cells. Time course studies showed that ORMDL3-deficient A549 and NHBE cells had an attenuated IL-6 and IL-8 response to poly I:C stimulation. A549 and NHBE cells over-expressing ORMDL3 released relatively more IL-6 and IL-8 following poly I:C stimulation. IL-17A exhibited a similar inflammatory response in ORMDL3 knockdown and overexpressing cells, but co-stimulation of poly I:C and IL-17A did not significantly enhance the IL-6 and IL-8 response. Transcript abundance of IFNB following poly I:C stimulation was not significantly altered by ORMDL3 knockdown or over-expression. Dampening of the IL-6 response by ORMDL3 knockdown was confirmed in HRV16 infected BEAS-2B and A549 cells.
\end{abstract}

Conclusions: ORMDL3 regulates the viral inflammatory response in airway epithelial cells via mechanisms independent of the TLR3 pathway.

Keywords: ORMDL3, Poly I:C, TLR3, Epithelial cell, Inflammatory response

*Correspondence: y.zhang@imperial.ac.uk

${ }^{1}$ National Heart and Lung Institute, Imperial College London,

London SW3 6LY, UK

Full list of author information is available at the end of the article

\section{Background}

Asthma is a syndrome defined by symptoms of reversible airway inflammation, intermittent wheeze and shortness of breath, and caused by a combination of genetic, epigenetic and environmental factors. Acute exacerbations are the major cause of morbidity and mortality from the disease and severe asthma remains difficult to treat due to lack of effective therapeutic options. In 2007, a 
genome-wide association study (GWAS) of asthma identified single nucleotide polymorphisms (SNPs) flanking ORMDL3 gene on chromosome 17 to be significantly associated with the disease [1]. This association then has subsequently been replicated in many studies worldwide, including a multi-ancestry global meta-analysis [2]. Chromosome 17 ORMDL3 locus is now recognised as the major predisposing factor for childhood-onset asthma. Significant increases in the number of wheezing illnesses have been observed in children with enhanced transcription genotypes at the 17q21 ORMDL3 locus.

Rhinoviruses are the most frequent pathogen associated with the symptoms of virial respiratory infections (VRI) [3] and early symptomatic human rhinovirus (HRV) infection is a major risk factor for subsequent asthma. HRV infection accounts for nearly two thirds of childhood asthma exacerbations [4]. Evidence now indicates that the association between the 17q21 ORMDL3 locus and childhood-onset asthma is also limited to those children with HRV-induced wheezing in early life [5], however the mechanism for this interaction remains unclear. We previously reported that ORMDL3 has multiple effects on host-pathogen interactions, stress responses and ubiquitination in cells. Importantly, ORMDL3 regulates the major HRV receptor, intercellular adhesion molecule 1 (ICAM1) during the IL- $1 \beta$ stimulated inflammatory response [6]. ORMDL3 was also found to promotes HRV replication in human epithelial cells [7]. Human ORMDL3 is a trans-membrane protein anchoring in the endoplasmic reticulum (ER). The ER is the site responsible for protein folding, synthesis of lipids and the storage of free calcium. ER stress can reduce the capacity of the ER for protein folding and thereby dictate cellular responses to inflammation. It interacts with the serine SPT enzyme complex in sphingolipid synthesis [8]. ORMDL3 facilitates the unfolded protein response to cellular stress by influencing sarcoplasmic/endoplasmic reticulum calcium ATPase (SERCA) and ER-mediated $\mathrm{Ca}^{2+}$ flux [9]. ORMDL3 also regulate endoplasmic reticulum (ER) stress, cermide and shingosine-1-sulfate levels that regulate cytokine release. ORMDL3 could work in multiple pathways in regulating HRV infections [10].

In this brief report, we investigated the role of ORMDL3 in the host inflammatory response to viral stimulation, as mimicked by polyinosinic-polycytidylic acid (poly I:C), in airway epithelial cells. Poly I:C is a synthetic analog of viral double-stranded RNA (dsRNA) and a mimic of viral infection. is recognized by toll-like receptor 3 (TLR3, also known as CD283) in a variety of cell types, including B-cells, macrophages, dendritic and epithelial cells $[11,12]$, triggering an innate immune response and recapitulating the major cellular, physiological and molecular changes characteristic of the host inflammatory response to viral infection. These include a loss of epithelial integrity, increased production of mucus and induction of inflammatory cytokines, most notably, the major mediator of host antiviral defence; interferon (IFN)- $B$ [13]. Recent evidence indicates that IL-17A and poly I:C act synergistically to induce production of chemo-attractants CXCL1 and IL-8 [14] and release of inflammatory cytokines [15] in bronchial epithelial cells. We established ORMDL3 knockdown and over-expression models in A549 epithelial and NHBE cells and stimulated the cells with either poly I:C or IL-17A alone or in combination. The inflammatory response measured over a 24-h time course for mono-stimulation experiments, and at $10 \mathrm{~h}$ for co-stimulation experiments through mRNA levels of key genes and protein levels of IL-6 and IL-8 in the supernatant. We finally tested the inflammatory cytokine release in ORMDL3 knockdown A549 and BEAS-2B epithelial cells infected by live human rhinovirus (HRV16).

\section{Methods \\ Cell culture and stimulations}

Human lung epithelial cell line A549 cells were purchased from American Type Culture Collection (ATCC). The cells were cultured in Dulbecco's Modified Eagle's Medium (DMEM) containing 10\% vol/vol (v/v) FBS and $2 \mathrm{mM}$ L-Glutamine. A549 cells were maintained in $150 \mathrm{~cm}^{2}$ (T150) flasks at $37{ }^{\circ} \mathrm{C}$ with $5 \% \mathrm{CO}_{2}$. The SV-40 immortalised human bronchial epithelial cell line BEAS2B was purchased from American Type Culture Collection (ATCC, Manassas, VA, USA), maintained in LHC-8 medium (Invitrogen, Paisley, UK) at $37{ }^{\circ} \mathrm{C}, 5 \% \mathrm{CO}_{2}$, and sub-cultured twice a week. Normal human bronchial epithelial (NHBE) cells were obtained from Lonza and cultured in human airway epithelial cell (hAEC) culture medium (Epithelix, Switzerland). Poly I:C was purchased from Sigma-Aldrich (cat P1530, UK). Recombinant human IL-17A was obtained from R\&D (cat 317-ILB050, UK). Following gene knockdown or over-expression and incubation with the respective culture medium in 24 well plates, A549 and NHBE cells were stimulated in triplicate with $10 \mu \mathrm{g} / \mathrm{ml}$ poly I:C per well or/with $100 \mu \mathrm{g} / \mathrm{ml}$ IL-17A per well.

\section{ORMDL3 knockdown and overexpression}

ORMDL3 gene knockdown was performed by using gene specific ORMDL3 siRNA ON-TARGETplus SMARTpool (Dharmacon Research Inc., Lafayette, CO). ONTARGETplus Non-targeting Pool (Dharmacon Research Inc., Lafayette, $\mathrm{CO}$ ) was used for control in the experiments. Transfection of 40,000 A549 cells each well was carried out with using Thermo Scientific DharmaFECT transfection reagent 4 . In total $25 \mathrm{nM}$ of siRNA in serum 
free medium (Sigma-Aldrich) was added to each well simultaneously and the cells were placed in a $\mathrm{CO}_{2}$ incubator at $37{ }^{\circ} \mathrm{C}$ for $48 \mathrm{~h}$. The medium of each cell was removed, cells were starved in serum free medium for $24 \mathrm{~h}$ and then stimulated with poly I:C or IL-17A. For construction of an ORMDL3 over-expression plasmid, we generated an ORMDL3 gene specific PCR from control cDNA (Clonetech, USA). The sequences for primers were: forward, CACCATGAATGTGGGCACAGCGCA CAGCGAG; reverse, TCAGTACTTATTGATTCCAAA AATC. The PCR product was then cloned to the pcDNATM3.1 directional TOPO ${ }^{\circledR}$ expression vector (Invitrogen) according to the manufacturer's protocol. Clones containing the correct insert were sequenced and verified. For the over-expression experiment $250 \mathrm{ng}$ of the $O R M D L 3$ over-expressing plasmid or control plasmid with $1 \mu$ lipofectamine ${ }^{\mathrm{TM}} 2000$ (Invitrogen) were used per well. Cells were cultured for $48 \mathrm{~h}$, then the medium was removed and serum free medium was added for $24 \mathrm{~h}$. Cells and supernatants were collected at 0, 6, 10, 16 and $24 \mathrm{~h}$ for time course studies. For co-stimulation of poly $\mathrm{I}: \mathrm{C}$ and IL-17A, samples were collected at $10 \mathrm{~h}$.

\section{Western blotting}

For ORMDL3 expression, whole-cell protein extracts were prepared by using the RIPA Lysis Buffer (Millipore). For each sample, $50 \mu \mathrm{g}$ of protein was separated by electrophoresis on $10 \%$ sodium dodecyl sulfate polyacrylamide gels (Invitrogen) and transferred to nitrocellulose membranes using the iBlot $^{\mathrm{TM}}$ DryBlotting device (Invitrogen) and iBlot $^{\mathrm{TM}}$ Transfer stacks. After blocking for $1 \mathrm{~h}$ with $5 \%$ milk, the blot was immersed with the first antibody (1: 500, Rabbit anti-human ORMDL3, ABGENT Cat no: AP10739c) and then detected using secondary antibodies conjugated to horseradish peroxidase (HRP) (1:4000; DAKO Cytomation) and enhanced chemi-luminescence GE Healthcare ECL/ECL Plus (Amersham) detection solutions.

\section{Enzyme-linked immunosorbent assays (ELISA)}

Cell-free supernatants were harvested and stored for further cytokine measurements. Human IL- 6 and IL-8 were measured using ELISA kits according to the manufacturer's instructions (PeproTech, US).

\section{Quantitative PCRs}

RNA was extracted from samples using the protocol and RNeasy Plus Mini Kit provided by Qiagen (Qiagen, UK). Extracted RNA was transcribed into complementary DNA (cDNA) using a High-Capacity cDNA Reverse Transcription Kit (Applied Biosystems, Lithuania). qPCRs were performed in QuantStudio 7 Flex RealTime PCR System with KAPA SYBR ${ }^{\circledR}$ FAST Universal
(Sigma-Aldrich). Forward and reverse primer sequences are: GAPDH, CACCATCTTCCAGGAGCGAG, CCTTCT CCATGGTGGTGAAGAC; ORDML3,TTGTGAGTGTCC CTGTCG, AGTGTAGAAGCTGGTGAGG; TLR3, AGA GTTGTCATCGAATCAAATTAAAG, AATCTTCCAATT GCGTGAAAA; TRIF, CCGGATCCCTGATCTGCTTG, ATGTCGAAGGCGCTAGGAAG; IFNB, GATTCATCT AGCACTGGCTGG, CTTCAGGTAATGCAGAATCC; IL1,TCTTTCTGGCTTAGAACAAAGGGGC, AGTAAA GGTAGCCCTTGTTTCCCCC; IL6, TACCCCCAGGAG AAGATTCC, GCCATCTTTGGAAGGTTCAG; IL8, ACT GAGAGTGATTGAGAGTGGAC, AACCCTCTGCAC CCAGTTTT.

\section{TLR3 transcript levels in microarray data from ORMDL3 knockdown A549 cells}

We examined TLR3 transcript levels in our previously published global gene profiling of ORMDL3 knockdown in A549 cells. The methods, protocols and analysis methods are described in [6].

\section{HRV16 infection in ORMDL3 knockdown A549 and BEAS-2B} cells

Human rhinovirus (HRV16) (ATCC ${ }^{\circledR} \mathrm{VR}-283^{\mathrm{TM}}$ ) was purchased from American tissue culture collection (Manassas, VA, USA), and propagated in Hela cells $\left(\mathrm{ATCC}^{\circledR} \mathrm{CRL}-1958^{\mathrm{TM}}{ }^{\text {) }}\right.$. A549 cells or BEAS-2B cells were transfected with ORMDL3 siRNA or scramble oligonucleotide as shown above and incubated for 4 days at $37^{\circ} \mathrm{C}, 5 \% \mathrm{CO}_{2}$. HRV16 (5 multiplicity of infection, MOI) was then applied to cells and incubated for $48 \mathrm{~h}$ at $33^{\circ} \mathrm{C}$, $5 \% \mathrm{CO}_{2}$. The cell-free supernatant was harvested and stored for IL- 6 and IL- 8 measurements. Human IL- 6 and IL-8 was measured using ELISA kits as shown above. IL-6 and IL-8 levels of non-infection cells were applied to be compared in each group.

\section{Statistical analysis}

Human IL-6 and IL-8 arithmetic means were compared by one-way analysis of variance [1]. All statistical analyses were performed, and graphs drawn using GraphPad Prism Version 5.01 software (GraphPad Software Inc, California, USA). Quantitative real-time PCR (qPCR) was conducted using QuantStudio 7 Flex Real-Time PCR System (Applied Biosystems). Measurements were ascertained in triplicate for each gene (ORDML3, TLR3, TRIF, $I F N B, I L 1, I L 6, I L 8)$, with GAPDH as the reference gene. The comparisons of $\triangle \triangle \mathrm{CT}$ of each experiment groups were performed with Student's $t$ tests. Data presented are averages \pm SEM (standard error of the mean). All statistical hypothesis tests were two tailed and a $P$ value of less than 0.05 was considered significant. 


\section{Results}

ORMDL3 does not impact baseline TLR3 expression in A549 cells

TLR3 transcript abundance data were available as part of a prior global gene expression time course study of ORMDL3 knockdown and IL-1 $\beta$ stimulation in A549 cells [6]. TLR3, as indexed by Affymetrix Human Gene 1.1 ST transcript cluster 8,098,611, showed no significant difference in baseline gene expression dependent on ORMDL3 knockdown status (control group expression 7.21 , knockdown group $7.48, P=5.60 \times 10^{-02}$ ). Looking across the time-course, TLR3 showed a small, nominally significant difference in response to IL-1 $\beta$ stimulation between control cells and cells deficient for ORMDL3 $\left(\mathrm{F}=3.27, P=2.31 \times 10^{-02}\right)$, though significance was not retained following a global $P$ value adjustment. The transcript abundance of TLR3 in ORMDL3 knockdown and control cells is listed in Table 1 . The results indicate that ORMDL3 may have a minor influence on TLR3 expression, detectable only under stimulation. To confirm absence of a baseline difference we used siRNA to silence $O R M D L 3$ and the $p c D N A 3.1-O R M D L 3$ plasmid to generate over-expression of ORMDL3 in A549 cells (Fig. 1a). We examined TLR3 transcript levels in the cells. The transcript abundance of TLR3 did not differ significantly between cells over-expressing or deficient for ORMDL3. Together these data suggest that ORMDL3 expression has no significant influence of the baseline expression of TLR3 in epithelial cells (shown in Fig. 1b, c).

\section{$O R M D L 3$ regulates the poly $\mathrm{I}: \mathrm{C}$-induced inflammatory response in A549 cells}

In order to determine whether poly I:C can induce an inflammatory response in human airway epithelial cells we stimulated A549 cells with poly I:C at concentrations of $0,1,1,5,10$ and $50 \mu \mathrm{g} / \mathrm{ml}$ for $10 \mathrm{~h}$. Poly I:C induced an inflammatory response in a dose dependent manner with supernatant IL-6 and IL-8 levels increasing with each poly I:C dosage increment (shown in Fig. 2a, b). We monitored the cell viability at each poly I:C dosage concentration. Poly I:C affected the cell viability at a concentration of $50 \mu \mathrm{g} / \mathrm{ml}$ (data not shown). We therefore applied a concentration of $10 \mu \mathrm{g} / \mathrm{ml}$ for all further experiments. In order to assess participation of ORMDL3 in the poly I:Cinduced inflammatory response we stimulated ORMDL3deficient and ORMDL3 over-expressing A549 cells with poly I:C for $10 \mathrm{~h}$. ORMDL3 knockdown resulted in a blunted inflammatory response to poly $\mathrm{I}: \mathrm{C}$, with relatively reduced IL-6 and IL- 8 release at $10 \mathrm{~h}(P<0.05$ respectively; Fig. 2c, d). Conversely ORMDL3 over-expression yielded an exaggerated inflammatory response, with poly I:C inducing relatively higher levels of IL- 6 and IL-8 release $(P<0.05$ respectively, Fig. $2 \mathrm{e}, \mathrm{f})$. These results confirm that poly I:C induces an inflammatory response in airway epithelial cells and identify $O R M D L 3$ as a factor regulating the magnitude of this response.

\section{Time course studies of poly I:C stimulation in A549 and NHBE cells}

To further explore the role of ORMDL3 in the poly I:C inflammatory response we set up 24-h time-course studies of poly I:C stimulation in A549 and NHBE cells deficient or over-expressing for ORMDL3. After a 48-h transfection cells were stimulated with poly I:C $(10 \mu \mathrm{g} /$ $\mathrm{ml})$ and supernatant samples collected at 0, 6, 10, 16 and 24 h. IL-6 and IL- 8 production in each condition was measured and compared to their respective controls. In A549 cells under conditions of ORMDL3 knockdown IL-6 levels were relatively reduced at all time-points except $0 \mathrm{~h}$ (baseline), with statistical significance achieved

Table 1 The TLR3 transcript levels in ORMDL3 knockdown A549 cells during IL1b stimulation

\begin{tabular}{|c|c|c|c|c|c|c|c|c|}
\hline Time points & Group & Avr. exp & Comparison & Fold change & Q value & Comparison & Fold change & $\mathrm{Q}$ value \\
\hline $\mathrm{Oh}$ & Control & 7.21 & - & & & - & & \\
\hline $2 \mathrm{~h}$ & Control & 7.40 & $2 \mathrm{~h} / 0$ control & 1.15 & 0.35 & - & & \\
\hline $4 \mathrm{~h}$ & Control & 7.38 & $4 \mathrm{~h} / 0$ control & 1.14 & 0.24 & - & & \\
\hline $8 \mathrm{~h}$ & Control & 7.10 & $8 \mathrm{~h} / 0$ control & 0.89 & 0.30 & - & & \\
\hline $10 \mathrm{~h}$ & Control & 6.98 & 10 h/0 control & 0.87 & 0.22 & - & & \\
\hline $\mathrm{Oh}$ & siRNA & 7.48 & - & & & O h siRNA/control & 1.24 & 0.27 \\
\hline $2 \mathrm{~h}$ & SiRNA & 7.15 & $2 \mathrm{~h} / 0$ siRNA & 0.78 & 0.03 & 2 h siRNA/control & 0.83 & 0.26 \\
\hline $4 \mathrm{~h}$ & siRNA & 7.62 & $4 \mathrm{~h} / 0$ siRNA & 1.06 & 0.59 & $4 \mathrm{~h}$ siRNA/control & 1.16 & 0.81 \\
\hline $8 \mathrm{~h}$ & siRNA & 7.19 & $8 \mathrm{~h} / 0$ siRNA & 0.81 & 0.06 & 8 h siRNA/control & 1.12 & 0.67 \\
\hline $10 \mathrm{~h}$ & siRNA & 7.17 & $10 \mathrm{~h} / 0$ siRNA & 0.79 & 0.06 & 10 h siRNA/control & 1.14 & 0.81 \\
\hline
\end{tabular}

Total $25 \mathrm{nM}$ ORMDL3 siRNA and control were transfected into A549 cells. RNAs were extracted from the cells after IL-1B (1 ng/ml) stimulations at 0, 2, 4, 8 and 10 h. Global gene profiling was performed by using Affymetrix Human Gene $1.1 \mathrm{ST}$ Arrays. $0 \mathrm{~h}, 2 \mathrm{~h}, 4 \mathrm{~h}, 8 \mathrm{~h}, 10 \mathrm{~h}$ indicate the sample collecting time after IL-1B stimulation Avr. Exp average expression; h: hour 


\section{ORMDL3 expression in knockdown and over expression}

\section{A549 cells}

$\beta$-actin

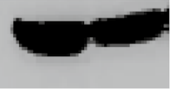

ORMDL3

a

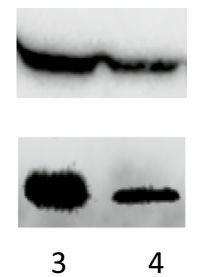

1. SIRNA_ORMDL3

2. Scramble control

3. pCDNA3.1_ORMDL 3

4. pcDNA3.1 control

TLR3 expression in ORMDL3 knockdown A549 cells

TLR3 expression in ORMDL3 over expression A549 cells
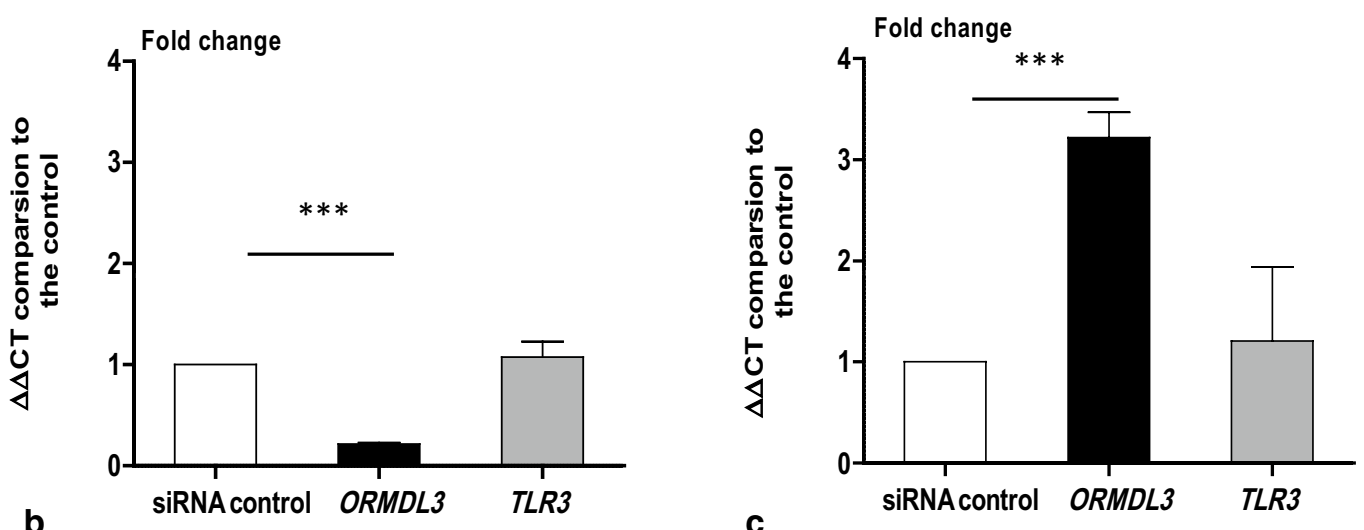

Fig.1 TLR levels in ORMDL3 knockdown and over-expressing A549 cells. A549 cells were seeded at 40,000 cell/per well in 24 well culture plate. a Western Blotting images of ORMDL3 expression in knockdown and over-expressing A549 cells. Full-length blots were presented in Additional file 1: Western Bollting Images of ORMDL3. b $25 \mathrm{nM}$ siRNA control and ORMDL3 siRNA were added into the cell culture medium. The cells were cultured for 48 h. c $250 \mathrm{ng} / \mathrm{ml}$ pcDNA3.1-ORMDL3 plasmid and control plasmid were added into the cell culture medium. Transcript levels of ORMDL3, TLR3 were quantified by qPCR and with GAPDH as internal control. $\triangle \triangle C T$ were compared with siRNA control samples. Student's $t$ test was calculated. ***Indicates $P<0.001$. Each group contained triplicated experiments

at $10 \mathrm{~h}$ only $(P<0.05)$. A consistent decrease in release from ORMDL3 knockdown cells was observed from 10 to $24 \mathrm{~h}$. IL-8 levels were also significantly decreased at $10 \mathrm{~h}(P<0.05)$ but showed no consistent trend at other time points (shown in Fig. 3a, b). For A549 cells overexpressing ORMDL3 IL-6 levels were relatively increased at $6 \mathrm{~h}$ but this difference achieved significance at $10 \mathrm{~h}$ only $(P<0.05$, shown in Fig. $3 \mathrm{c}, \mathrm{d})$. For NHBE cells, ORMDL3-deficient cells released significantly less IL-6 at 10 and $16 \mathrm{~h}$ after poly I:C stimulation $(P<0.05)$. IL-8 levels were also significantly lower at 6 and $16 \mathrm{~h}$ time-points compared to control $(P<0.05)$ (shown in Fig. 3e, f). For ORMDL3 over expressing NHBE cells, whilst both IL-6 and IL-8 levels were elevated relative to control at all time points, these differences did not achieve statistical significance (shown in Fig. 3g, h).

Our results confirm a bidirectional influence of $O R M D L 3$ on the poly I:C inflammatory response in epithelial cells. Silencing of ORMDL3 blunts the inflammatory response whilst over-expression increases its magnitude. The impact of ORMDL3 manipulation on the poly I:C response was not however, as large as its impact on the IL- $1 \beta$ response as reported in our previous experiments [6].

\section{Co-stimulation of poly I:C and IL-17A in ORMDL3 knockdown and over-expressing A549 cells}

The Th17 cytokine IL-17A can enhance the poly I:C inflammatory response in epithelial cells [15]. We therefore investigated the impact of ORMDL3 knockdown and over-expression under conditions of poly I:C and IL-17A co-stimulation in A549 cells.

In ORMDL3-deficient cells, as expected, poly I:C induced lower IL-6 levels at $10 \mathrm{~h}$ relative to control $(P<0.05)$. Likewise, IL-17A also induced lower IL-6 levels in ORMDL3-deficient cells relative to control $(P<0.01)$. Whilst co-stimulation of poly I:C with IL-17A enhanced the IL-6 response in control cells $(P<0.001)$, this response did not differ significantly between control and ORMDL3 knockdown cells $(P=0.16$, shown in Fig. 4a). 


\section{IL-6 Level}

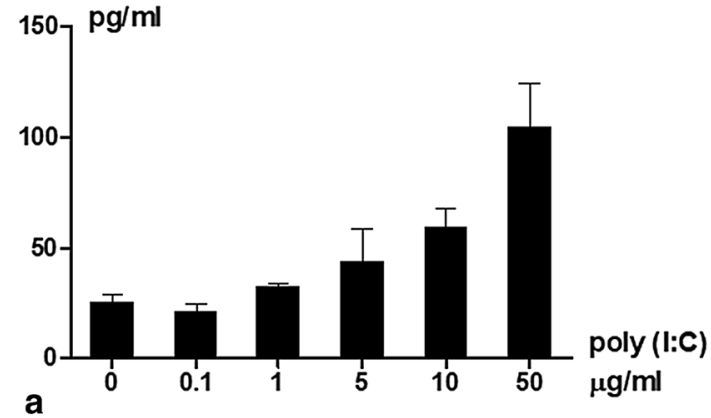

IL-6 Level

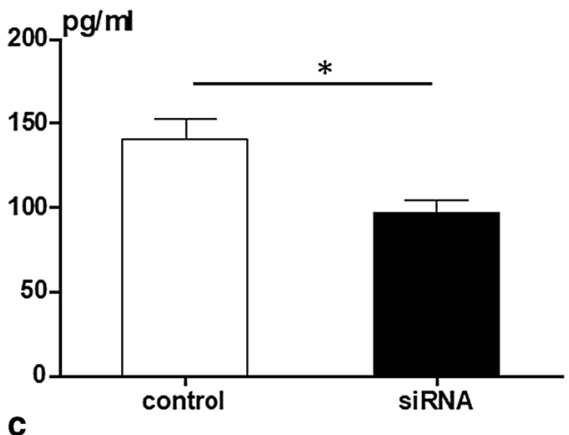

IL-6 Level

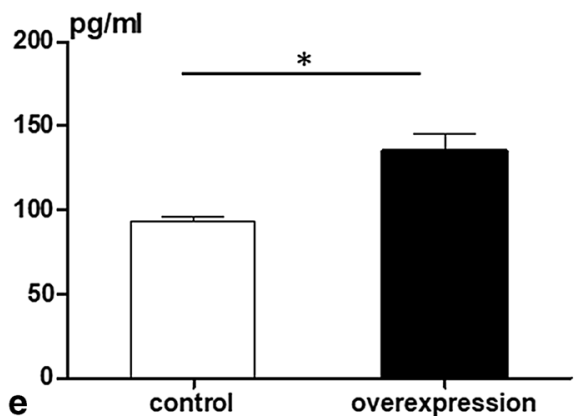

IL-8 Level

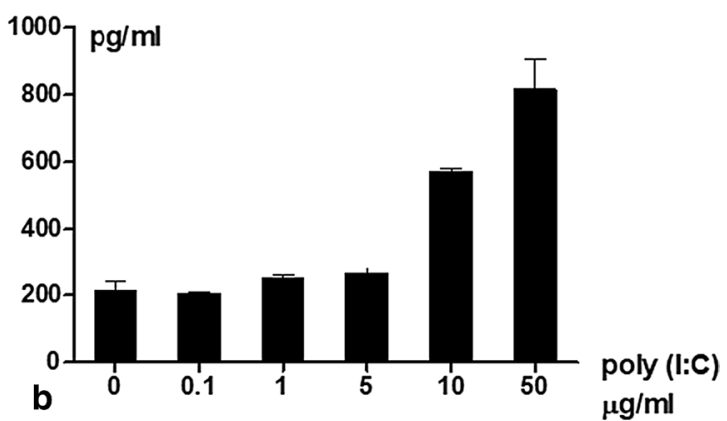

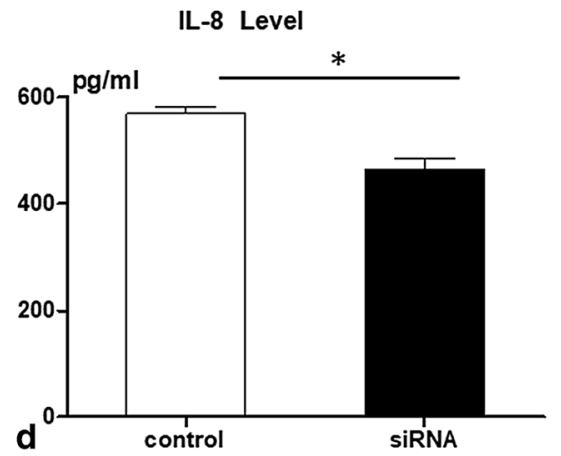

IL-8 Level

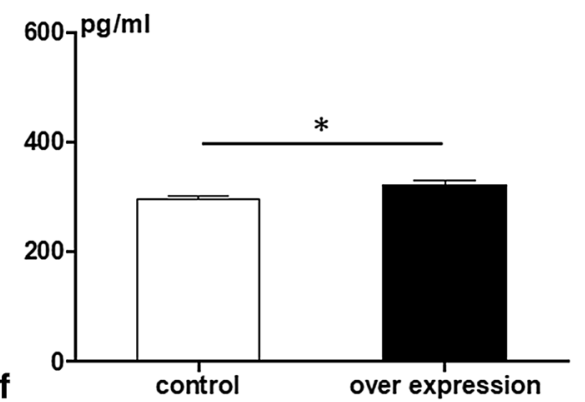

Fig.2 Inflammatory response induced by poly I:C in A549 cells. A549 cells were seeded with full medium for $24 \mathrm{~h}$, then changed to serum-free medium for 1 day. Poly I:C was added to the wells at different concentrations. Supernatants were collected for measurements for IL-6 and IL8 levels after $10 \mathrm{~h}$. a The IL-6 levels of poly I:C titration, $\mathbf{b}$ the IL-8 levels of poly I:C titration. c The IL-6 levels in ORMDL 3 knockdown cells after poly I:C stimulation for $10 \mathrm{~h}$. d The IL-8 levels after ORMDL3 knockdown cells after poly I:C stimulation for $10 \mathrm{~h}$. e The IL-6 levels in PCDNA3.1-ORMDL3 over-expressing cells after poly $\mathrm{l}: \mathrm{C}$ stimulation for $10 \mathrm{~h}$. f The IL-8 levels after PCDNA3.1-ORMDL3 over-expressing cell after poly I:C stimulation for $10 \mathrm{~h}$.

Similarly, whilst both poly I:C and IL-17A yielded lower magnitude IL-8 responses in ORMDL3 knockdown cells relative to control when applied independently $(P<0.05)$, no difference in IL-8 levels was seen between control and ORMDL3 knockdown cells under poly I:C and IL-17A co-stimulation ( $P=0.12$, shown in Fig. 4 b). In ORMDL3 over expressing cells both poly I:C and IL-17A led to an elevation in IL-6 levels relative to control when applied independently $(P<0.05)$, however no difference was seen between control and ORMDL3 over expressing cells under conditions of co-stimulation $(P=0.79$, shown in Fig. 4c). Similarly, both poly I:C stimulation and IL17A stimulation yielded a relatively larger IL-8 response in ORMDL3 over-expressing cells relative to control when applied independently $(P<0.05)$, however no difference was observed between control and ORMDL3 


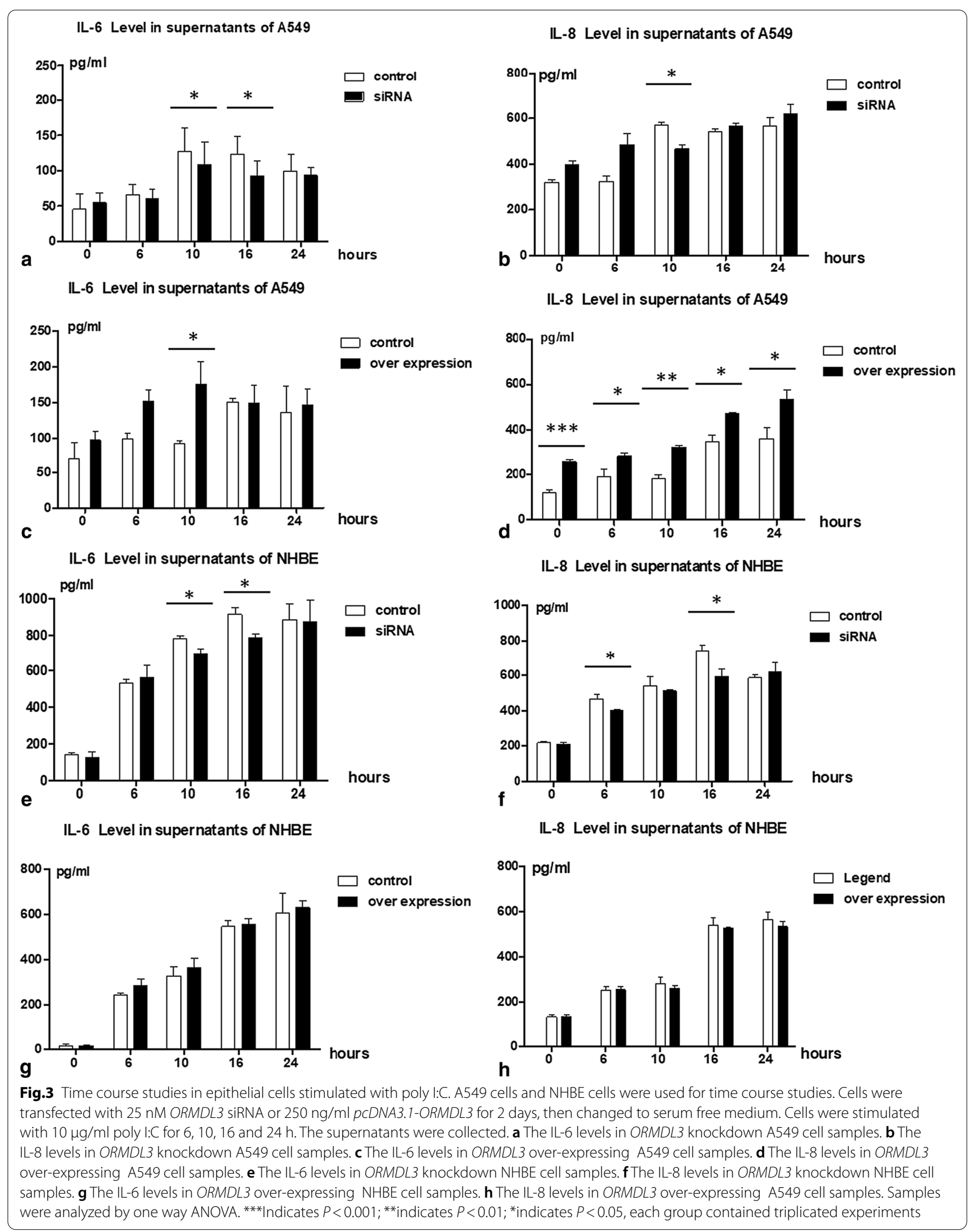


IL-6 levels in supernatants of A549 cells

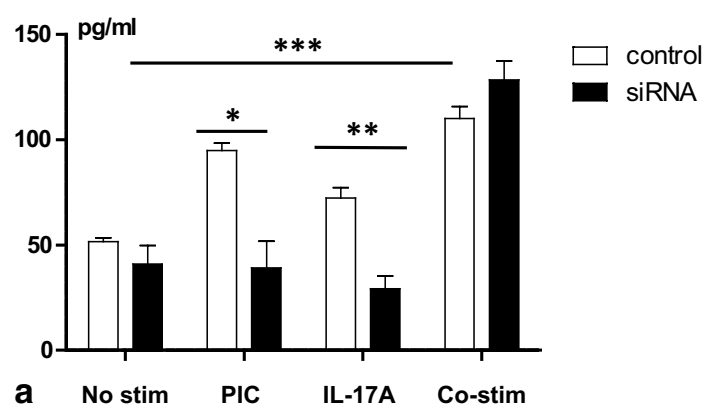

IL-6 levels in supernatants of A549 cells

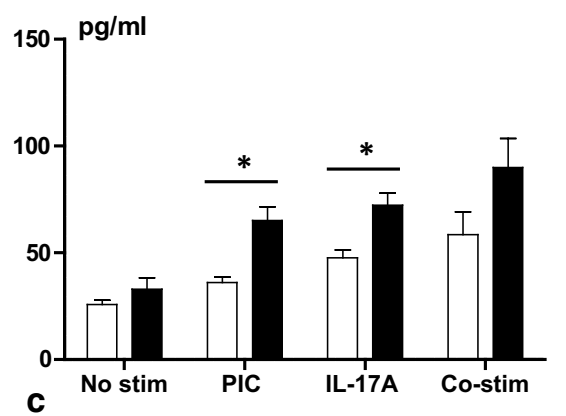

IL-8 levels in supernatants of A549 cells

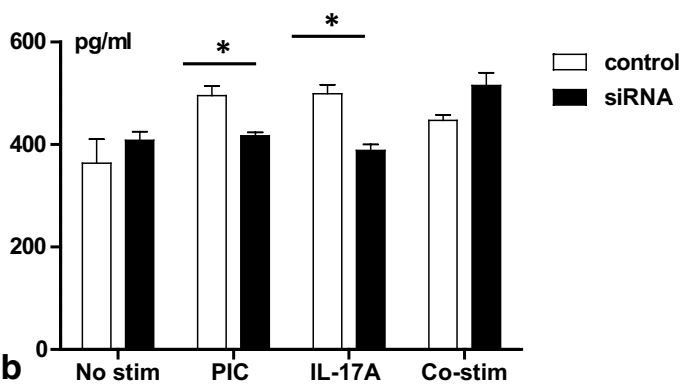

IL-8 levels in supernatants of A549 cells

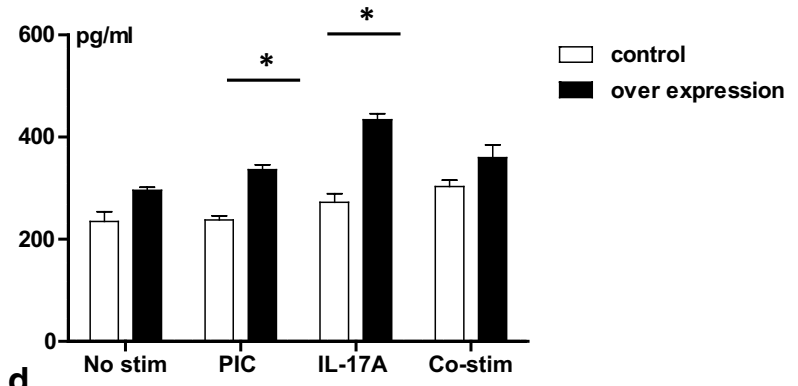

Fig.4 Co-stimulation with poly I:C and IL-17A in A549 cells. A549 cells were used for co-stimulations with $10 \mu \mathrm{g} / \mathrm{ml} \mathrm{poly}$ I:C and $100 \mu \mathrm{g} / \mathrm{ml} \mathrm{IL}-17 \mathrm{~A}$ for $10 \mathrm{~h}$ in ORMDL3 knockdown and ORMDL3 over-expression A549 cells for $10 \mathrm{~h}$. a The IL-6 levels in ORMDL3 knockdown A549 cell samples. b The IL-8 levels in ORMDL3 knockdown A549 cells. c IL-6 levels in ORMDL3 over-expressing A549 cells. d IL-8 levels in ORMDL3 over-expressing A549 cell samples. Samples were analyzed by one way ANOVA. ${ }^{* *}$ Indicates $P<0.001$; ${ }^{*}$ indicates $P<0.01$; *indicates $P<0.05$, each group contained triplicated experiments. PIC: poly I:C, Co-stim: co-stimulation of poly I:C and IL-17A, No-stim: no-stimulation

over expressing cells under conditions of co-stimulation $(P=0.46$, shown in Fig. $4 d)$.

These results show that ORMDL3 influences the inflammatory response induced by IL-17A and poly I:C in epithelial cells. Manipulation of ORMDL3 does not however, influence the response to co-stimulation, suggesting that ORMDL3 may have different, non-additive roles in the signal transduction of TRL and Th17 pathways in epithelial cells.

\section{The transcript levels of TLR3, IFNB, TRIF, IL1, IL6 and IL8} in ORMDL3 knockdown and over-expressing A549 cells after poly I:C stimulation

To further delineate the role of ORMDL3 we analysed the abundance of transcripts responsible for TLR3 signalling and the inflammatory response in A549 cells deficient for, or over-expressing ORMDL3 at baseline, and following $10 \mu \mathrm{g} / \mathrm{ml}$ poly I:C stimulation for $24 \mathrm{~h}$. IFN $\beta$ is type I class of interferon and is important for defence against viral infections. TRIF encodes an adaptor protein containing a toll/interleukin-1 receptor homology domain, which is an intracellular signalling domain that mediates protein-protein interactions between the TLRs and signal-transduction components. It specifically interacts with TLR3, but not with other TLRs, and this association mediates dsRNA induction of IFN $\beta$ through activation of nuclear factor kappa-B (NF- $\mathrm{KB})$ during an antiviral immune response. We investigated the transcript levels of TLR3, IFNB and TRIF and the inflammatory cytokine genes IL1, IL6 and IL8. In ORMDL3 knockdown A549 cells at baseline, transcript levels of TLR3, IFNB, TRIF, IL1, IL6 and IL8 showed no significant difference (shown in Fig. 5a). In ORMDL3 over-expressing cells at baseline the transcript levels of TRIF and ILI were significantly raised relative to the control group $(P<0.01, P<0.05$ respectively, shown in Fig. 5b). We then compared transcript levels at $24 \mathrm{~h}$ after stimulation. Surprisingly, we found that poly I:C significantly down regulated the TLR pathway genes IFNB and TRIF, and up regulated the cytokine genes IL1, IL6 and IL8 both after $24 \mathrm{~h}(P<0.001$ respectively, data not shown). Next, we compared the transcript levels in cells deficient for, or over-expressing ORMDL3 at $24 \mathrm{~h}$ following stimulation. For ORMDL3 knockdown cells, TRIF transcript levels were significantly lower than the control group $(P<0.001)$ (shown in Fig. 5c). For ORMDL3 over expressing cells, although 
The transcript levels in ORMDL3 knockdown A549 cells

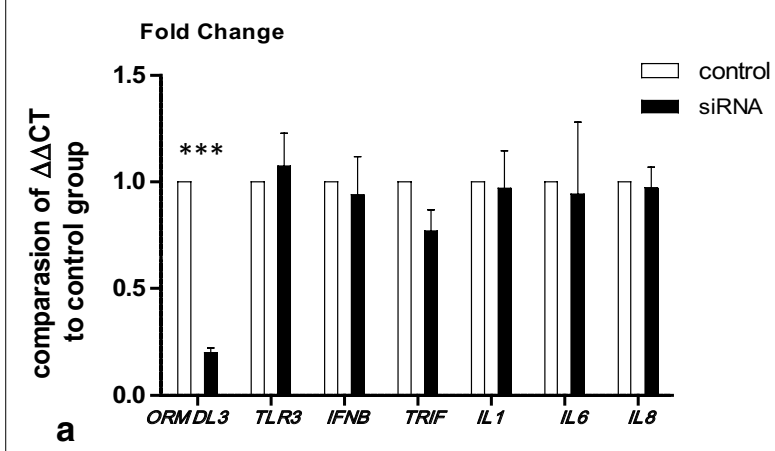

The transcript levels in ORMDL3 knockdown A549 cells after 24 hours poly I:C stimulation

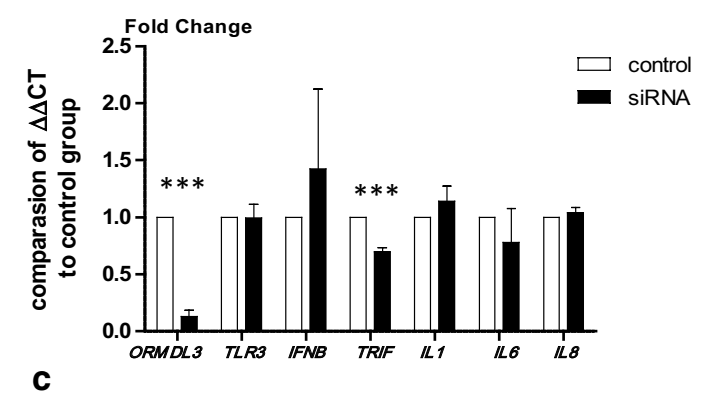

The transcript levels in ORMDL3 overexpression A549 cells

Fold Change

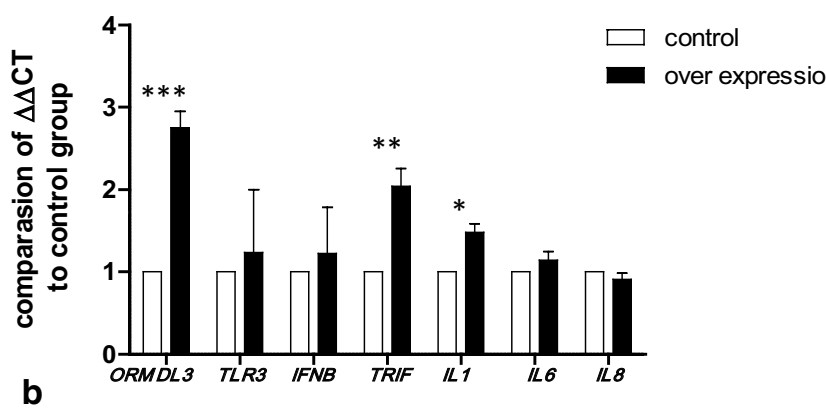

The transcript levels in ORMDL3 over expression in A549 cells after 24 hours poly I:C stimulation

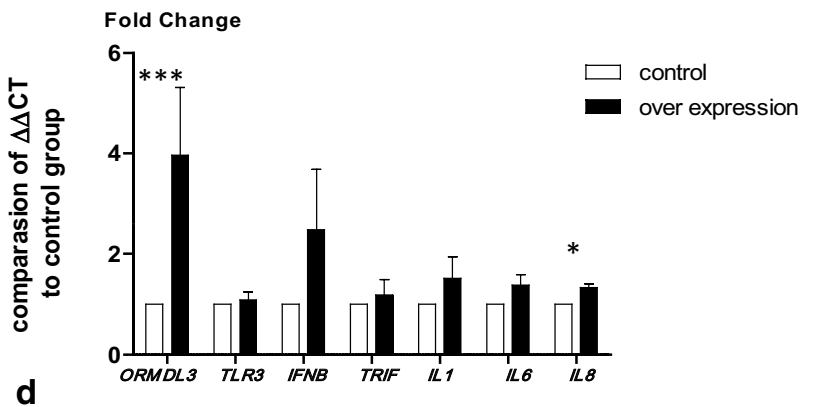

Fig. 5 Gene transcript levels in ORMDL3 knockdown and over-expression before and after poly l:C stimulation. ORMDL3 knockdown or over-expression A549 cells and NHBE cells were stimulated with $10 \mu \mathrm{g} / \mathrm{ml}$ poly l:C for $24 \mathrm{~h}$. RNAs were extracted and gPCRs were performed. a Gene transcript levels at ORMDL3 knockdown cells and control cells. b Gene transcript levels at ORMDL3 over-expressing cells and control cells. c Transcript levels at ORMDL3 knockdown cells and control cells after $24 \mathrm{~h}$ stimulation with poly I:C. $\mathbf{d}$ Gene transcript levels in ORMDL3 over-expressing cells and control cells after $24 \mathrm{~h}$ stimulations with poly l:C. ${ }^{* *}$ indicates $P<0.001$; ${ }^{*}$ indicates $P<0.01$; ${ }^{*}$ indicates $P<0.05$, each group contained triplicated experiments

transcript levels for all genes were higher than the control group, only the $I L 8$ transcript achieved significance $(P<0.05)$ (shown in Fig. 5d). The results show that whilst $O R M D L 3$ did not significantly influence transcript levels of TLR3 and IFNB after poly I:C stimulation, it did impact transcript levels of TRIF and several cytokine genes.

\section{The inflammatory response in ORMDL3 knockdown epithelial cells after HRV-16 infection}

In order to determine whether ORMDL3 knockdown in epithelial cells also influences the inflammatory response to live viral infection, we silenced ORMDL3 in A549 and BEAS-2B epithelial cells and infected these cells with HRV16, one of the most common rhinoviruses. We then evaluated the effects of knockdown on HRV16-induced IL-6 and IL-8 release as compared to the non-infection control in each group. Basal levels of IL- 6 and IL-8 in control A549 cells transfected with scramble oligo were
$0.59 \pm 0.37 \mathrm{pg} / \mathrm{mL}$ and $5.2 \pm 0.20 \mathrm{ng} / \mathrm{mL}$, respectively, and HRV16 markedly induced IL-6 $(0.59 \pm 0.37 \mathrm{pg} /$ $\mathrm{mL})$ and only weakly induced IL-8 $(5.2 \pm 0.20 \mathrm{ng} / \mathrm{mL})$. In contrast, $O R M D L 3$ knockdown cells did not enhance IL-6 and IL-8 production after HRV16 inoculation (IL-6 [HRV16/no-inoculation]: $2.2 \pm 0.89 / 2.7 \pm 2.3 \mathrm{pg} / \mathrm{mL}$; IL-8 [HRV16/no-inoculation]: $6.2 \pm 0.12 / 6.8 \pm 0.46 \mathrm{pg} / \mathrm{mL}$ ). Basal levels of IL- 6 and IL-8 in control BEAS-2B cells transfected with scramble oligo were $2.8 \pm 0.11 \mathrm{pg} / \mathrm{mL}$ and $16.4 \pm 2.6 \mathrm{pg} / \mathrm{mL}$, respectively, and HRV16 markedly induced IL-6 $(246 \pm 31.8 \mathrm{pg} / \mathrm{mL})$ and IL-8 $(239 \pm 49.2 \mathrm{pg} /$ $\mathrm{mL})$. In contrast, in ORMDL3 knockdown cells, HRV16 induced IL- 6 and IL-8 production but the induction levels are relatively lower than those in control cells (IL-6 [HRV16/no-inoculation]: $231 \pm 28.8 / 4.1 \pm 1.2 \mathrm{pg} / \mathrm{mL}$; IL-8 [HRV16/no-inoculation]: $156 \pm 45.5 / 17.0 \pm 3.0 \mathrm{pg} /$ $\mathrm{mL})$. To demonstrate the difference clearer, we calculated the induction ratio [HRV16/non-inoculation] in each 
cytokine. As shown in Fig. 6, HRV16-dependent induction of IL-6 compared with base line was significantly lower in ORMDL3 knocked-down A549 cells $(P<0.05)$ and also bronchial epithelial cell line BEAS-2B $(P<0.05)$. For IL-8, levels were also somewhat reduced in the context of ORMDL3 knockdown, but this did not achieve statistical significance in either A549 cells $(P=0.20)$ or BEAS-2B cells $(P=0.13)$. These data are broadly consistent with our observations from the viral mimic poly I:C and together confirm that ORMDL3 is involved in HRV16-dependent cytokine production as well as poly I:C dependent cytokine release.

\section{Discussion}

Viral respiratory infections show robust association with asthma exacerbations in the community. Human rhinovirus, in particular, is the predominant virus associated with asthma attacks [16], although other viral pathogens such as RSV and influenza may also contribute to the disease $[17,18]$.

In this report, we generated ORMDL3 knockdown and $O R M D L 3$ over-expressing epithelial cells and investigated the inflammatory response after administration of poly I:C. Knockdown of ORMDL3 and overexpression had little influence of TLR3 transcript levels in A549 cells. However, in both A549 and NHBE cells, knockdown of ORMDL3 led to a blunted poly I:Cinduced inflammatory response. Conversely ORMDL3 over-expression led to an enhanced poly I:C-induced inflammatory response in these cells. The reduction of cytokine release by ORMDL3 knockdown was also confirmed in HRV16-infected cells. Thus, the results suggest a key role for ORMDL3 in the host response to viral infection.



BEAS-2B cells

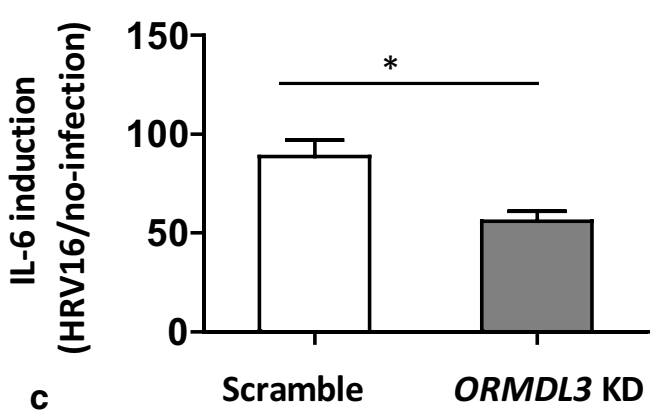

A549 cells

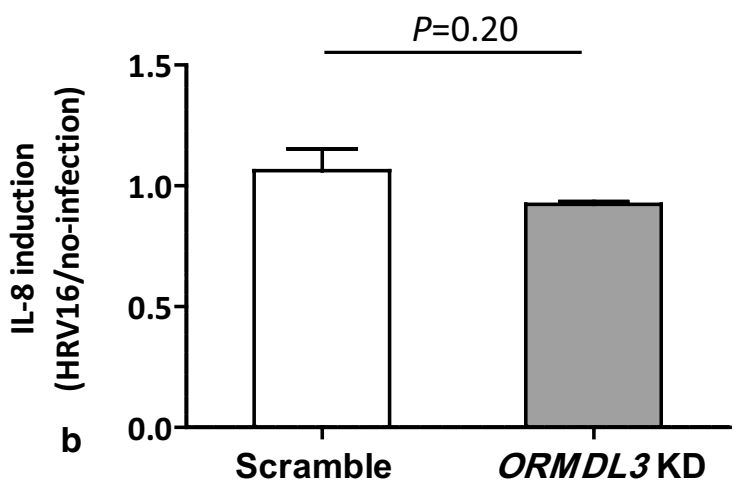

BEAS-2B cells

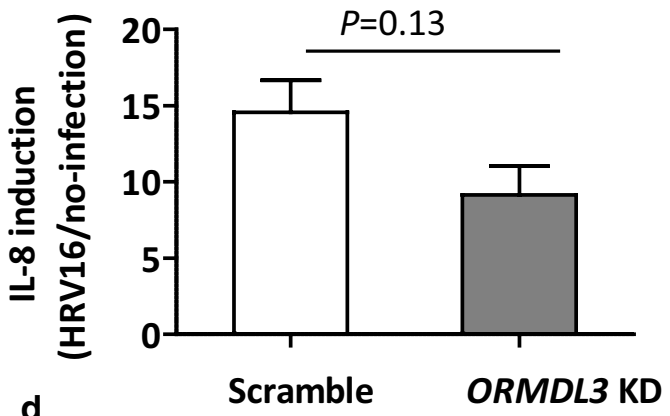

Fig.6 IL-6 and IL-8 levels in ORDML3 knockdown A549 and BEAS-2B epithelial cells after HRV-16 infection. ORMDL3 knockdown A549 and BEAS-2B cells were infected with HRV-16 (5 MOI) and incubated for $48 \mathrm{~h}$ at $33^{\circ} \mathrm{C}, 5 \% \mathrm{CO}_{2}$. The cell-free supernatant was harvested and both IL-6 and IL-8 were measured. IL-6 and IL-8 levels in non-infection cells were utilized as comparison in each group. a IL-6 induction levels in ORMDL3 A549 cells after HRV-16 infection. b IL-8 induction levels in ORMDL3 A549 cells after HRV-16 infection. c IL-6 induction levels in ORMDL3 BEAS-2B cells after HRV-16 infection. d IL-8 induction levels in ORMDL3 BEAS-2B cells after HRV-16 infection. *Indicates $P<0.05$, each group contained triplicated experiments excepting experiment $A$ had six experiments 
The IL-23/Th17 pathway is a central component of cellular immunity and IL-17A is a signature cytokine of this pathway [19]. IL-17A is widely reported to regulate chronic inflammatory diseases, including respiratory diseases such as asthma [20]. We show here that IL-17A induces an inflammatory response in epithelial cells, and that $O R M D L 3$ regulates this response. In this report, we did not observe an impact of ORMDL3 on the inflammatory response to combined stimulations of poly I:C and IL-17A; suggesting that ORMDL3 participates in multiple pathways in epithelial cells.

Multi-transcript profiling showed that overexpression of ORMDL3 up-regulates TRIF, suggesting regulation of the inflammatory response through the NF- $\kappa B$ pathway. Following poly I:C stimulation control cells showed significant decreases in the transcript levels of IFNB and TRIF, and increases in the cytokines IL1, IL8 and IL6, indicating that poly $\mathrm{I}: \mathrm{C}$ is a potent inflammatory inducer. Knockdown of ORMDL3 blunted the TRIF response to poly I:C, confirming that ORMDL3 works in the NF- $\mathrm{kB}$ pathway [6]. Our results indicate that $O R M D L 3$ is an important regulator of the inflammatory response to poly $\mathrm{I}: \mathrm{C}$ in epithelial cells, and that this impact may not be restricted to the TLR3 signalling pathway-since we did not observe the significant differences in the expression of IFNB between ORMDL3 knockdown and control cells, or between ORMDL3 over-expressing cells and control cells.

Viral respiratory tract infections are an established risk factor for asthma and can cause symptom relapse or exacerbations. The mechanisms underpinning these features are not known. Viral infection could contribute to asthmatic symptoms and/or change airway physiology [21]. TLRs transduce the infection signal through MAPKs, NF- $k B$, and IRF3, which induce the transcription of proinflammatory cytokines and type I interferon [22]. TRIF signalling also has both protective and pathologic roles in several chronic inflammatory disease conditions [23].

Toll-like receptors (TLRs) play important roles in the induction of innate antiviral immune responses to viral infections as well as in in development, homeostasis and injury repair [24]. A total of ten TLRs have been identified in humans. Of these, TLR3, first characterized as a regulator of anti-viral responses $[25,26]$. TLR3 is assembled in the endoplasmic reticulum, from where it is recruited to endosomes by the transmembrane protein UNC93B1 [27]. TLR3 directly recruits TRIF to its TIR domain to initiate signalling, leading to the activation of the serine/threonine kinase TBK-1, which in turn phosphorylates interferon regulatory factor 3 (IRF3) [24]. TLR3 signalling via TRIF also activates
NF-kB [28]. As such, treatment of wounded skin with poly I:C; a dsRNA mimic, significantly reduces recovery time in both humans and mice [29]. In this study, we did not observe that ORMDL3 directly influenced $T L R 3$ and IFNB transcript level after poly I:C stimulation, but it regulated transcript levels of TRIF and several cytokine genes. ORMDL3 may work in multiple signaling pathways to regulating viral infection through ER stress, sphingolipid metabolism, glycolysis and other mechanisms [10].

In this report, we found ORMDL3 knockdown epithelial cells release less IL- 6 after HRV16 infection, consistently confirm the results from poly I:C stimulations in ORMDL3 knockdown cells. In an experiment with HRV14, another HRV strain used ICAM-1 as receptor for entrance of epithelial cells, IL- 6 and IL-8 levels were found to increase by the infection [30]. HRV induced the cytokines release may partly activate an NF-kB-dependent transcriptional activation pathway [31].

This is the first report on the role of ORMDL3 in the poly I:C stimulation response in epithelial A549 and NHBE cells. We recognise several limitations. Firstly, including use of a restricted $(24 \mathrm{~h})$ time course following poly I:C stimulation, and a synthetic mimic of viral infection. Secondly, HRV16 is less infectious to A549 and BEAS2B cells in monolayer cell sheet underneath culture media compare with primary epithelial cells, and high level of inoculum, which is not relevant to the clinical condition, was required to induce IL- 6 and IL-8. HRV16 does not replicate well in these cells compared with primary epithelial cells, either. To solve these problems, we will use air-liquid interface cultured human bronchial epithelium, which is known to be well infected with HRV16 [32], The viral attachment, replication and inflammatory response of HRV16 infection for ORMDL3 knockdown epithelial cells need to be future in-depth investigation. Nevertheless, the observations presented here have significant clinical implications, and warrant further investigation focusing on the precise mechanisms through which $O R M D L 3$ moderates the inflammatory response to viral infection.

\section{Conclusions}

ORMDL3 significantly influences release of cytokines following poly I:C stimulation in airway epithelial cells. $O R M D L 3$ regulates the poly I:C-induced inflammatory response via mechanisms independent of the TLR3 pathway. 


\begin{abstract}
Abbreviations
ORMDL3: Oroscomucoid 3; poly l:C: Polyinosinic-polycytidylic acid; dsRNA: Double-stranded RNA; TLR3: Toll-like receptor 3; NHBE cells: Normal human bronchial epithelial cells; IFNB: Interferon-ß; HRV: Human rhinovirus; ICAM1: Intercellular adhesion molecule 1; RSV: Respiratory syncytial virus; ER: Endoplasmic reticulum; SPT: Palmitoyltransferase; SERCA: Sarcoplasmic/endoplasmic reticulum calcium ATPase; MOI: Multiplicity of infection; NF-kB: Nuclear factor kappa-B.
\end{abstract}

\section{Supplementary Information}

The online version contains supplementary material available at https://doi. org/10.1186/s12890-021-01496-5.

Additional file 1. Western Blotting Images of ORMDL3.

\section{Acknowledgements}

$\mathrm{YZ}$ is an Asmraley Lecturer at National Heart and Lung Institute of Imperial College London. We are grateful to Drs. Matthew Coats and Daniel Brookes (both Pulmocide Ltd.) for assistance in optimisation of HRV16 infection in vitro study.

\section{Authors' contributions}

Y.Z. designed and supervised the overall study. G.L., Y.L., K.F. designed individual experimental components and performed all the experimental work. K.I. conducted HRV16-infection assay. S.W.O., M.M., and W.C. supervised the gene expression experiments and interpretation of the experimental results. Every author contributed the final version of the manuscript. All authors read and approved the final manuscript.

\section{Funding}

This work was supported by the Wellcome Trust for cell culture and research reagents and by the Asmarley Foundation for laboratory consumables.

\section{Availability of data and materials}

All data are available from the corresponding author on reasonable request.

\section{Declarations}

\section{Ethics approval and consent to participate}

No ethics approval is required for research on human epithelial cells.

\section{Consent for publication}

Not applicable.

\section{Competing interests}

There are no known conflicts of interest arising from this research.

\section{Author details}

${ }^{1}$ National Heart and Lung Institute, Imperial College London, London SW3 6LY, UK. ${ }^{2}$ Pulmocide Ltd., London WC2A 1AP, UK.

Received: 8 December 2020 Accepted: 6 April 2021

Published online: 17 May 2021

\section{References}

1. Moffatt MF, Kabesch M, Liang L, Dixon AL, Strachan D, Heath S, Depner $M$, von Berg A, Bufe A, Rietschel E et al. Genetic variants regulating ORMDL3 expression contribute to the risk of childhood asthma. Nature. 2007:448(7152):470-73.

2. Demenais F, Margaritte-Jeannin P, Barnes KC, Cookson WOC, Altmuller J, Ang W, Barr RG, Beaty TH, Becker AB, Beilby J, et al. Multiancestry association study identifies new asthma risk loci that colocalize with immunecell enhancer marks. Nat Genet. 2018;50(1):42-53.

3. Greenberg SB. Respiratory consequences of rhinovirus infection. Arch Intern Med 2003;163(3):278-84.
4. Johnston SL, Pattemore PK, Sanderson G, Smith S, Lampe F, Josephs L, Symington $\mathrm{P}, \mathrm{O}^{\prime}$ Toole S, Myint SH, Tyrrell DA, et al. Community study of role of viral infections in exacerbations of asthma in 9-11 year old children. BMJ 1995;310(6989):1225-29.

5. Caliskan M, Bochkov YA, Kreiner-Moller E, Bonnelykke K, Stein MM, Du G Bisgaard H, Jackson DJ, Gern JE, Lemanske RF Jr, et al. Rhinovirus wheezing illness and genetic risk of childhood-onset asthma. N Engl J Med. 2013;368(15):1398-407.

6. Zhang Y, Willis-Owen SAG, Spiegel S, Lloyd CM, Moffatt MF, Cookson W. The ORMDL3 asthma gene regulates ICAM1 and has multiple effects on cellular inflammation. Am J Respir Crit Care Med. 2019;199(4):478-88.

7. Liu Y, Bochkov YA, Eickhoff JC, Hu T, Zumwalde NA, Tan JW, Lopez C, Fichtinger PS, Reddy TR, Overmyer KA, et al. Orosomucoid-like 3 supports rhinovirus replication in human epithelial cells. Am J Respir Cell Mol Biol. 2020;62(6):783-92.

8. Breslow DK, Collins SR, Bodenmiller B, Aebersold R, Simons K, Shevchenko A, Ejsing CS, Weissman JS. Orm family proteins mediate sphingolipid homeostasis. Nature. 2010;463(7284):1048-53.

9. Cantero-Recasens G, Fandos C, Rubio-Moscardo F, Valverde MA, Vicente $\mathrm{R}$. The asthma-associated ORMDL3 gene product regulates endoplasmic reticulum-mediated calcium signaling and cellular stress. Hum Mol Genet. 2010;19(1):111-21.

10. Ito $K$, Zhang Y. The roles of ORMDL3 in rhinovirus infections. Am J Respir Cell Mol Biol; 2020.

11. Li YG, Siripanyaphinyo U, Tumkosit U, Noranate N, An A, Pan Y, Kameoka M, Kurosu T, Ikuta K, Takeda N, et al. Poly (I:C), an agonist of toll-like receptor-3, inhibits replication of the Chikungunya virus in BEAS-2B cells. Virol J. 2012;9:114.

12. Royer PJ, Henrio K, Pain M, Loy J, Roux A, Tissot A, Lacoste P, Pison C, Brouard S, Magnan A, et al. TLR3 promotes MMP-9 production in primary human airway epithelial cells through Wnt/beta-catenin signaling. Respir Res. 2017;18(1):208.

13. Field AK, Lampson GP, Tytell AA, Nemes MM, Hilleman MR. Inducers of interferon and host resistance, IV. Double-stranded replicative form RNA (MS2-Ff-RNA) from E. coli infected with MS2 coliphage. Proc Natl Acad Sci USA. 1967;58(5):2102-8.

14. Hartupee J, Liu C, Novotny M, Li X, Hamilton T. IL-17 enhances chemokine gene expression through mRNA stabilization. J Immunol. 2007;179(6):4135-41.

15. Matsuzaki H, Mikami Y, Makita K, Takeshima H, Horie M, Noguchi S, Jo T, Narumoto O, Kohyama T, Takizawa H, et al. Interleukin-17A and toll-like receptor 3 ligand poly $(\mathrm{I}: \mathrm{C})$ synergistically induced neutrophil chemoattractant production by bronchial epithelial cells. PLoS ONE. 2015;10(10):e0141746.

16. Johnston NW, Johnston SL, Duncan JM, Greene JM, Kebadze T, Keith PK, Roy M, Waserman S, Sears MR. The september epidemic of asthma exacerbations in children: a search for etiology. J Allergy Clin Immunol. 2005;115(1):132-8.

17. Sigurs N, Aljassim F, Kjellman B, Robinson PD, Sigurbergsson F, Bjarnason R, Gustafsson PM. Asthma and allergy patterns over 18 years after severe RSV bronchiolitis in the first year of life. Thorax. 2010;65(12):1045-52.

18. Veerapandian R, Snyder JD, Samarasinghe AE. Influenza in asthmatics: for better or for worse? Front Immunol. 1843;2018:9.

19. Gaffen SL, Jain R, Garg AV, Cua DJ. The IL-23-IL-17 immune axis: from mechanisms to therapeutic testing. Nat Rev Immunol. 2014;14(9):585-600.

20. Linden A, Dahlen B. Interleukin-17 cytokine signalling in patients with asthma. Eur Respir J. 2014:44(5):1319-31.

21. Oliver BG, Robinson P, Peters M, Black J. Viral infections and asthma: an inflammatory interface? Eur Respir J. 2014;44(6):1666-81.

22. Akira S, Takeda K. Toll-like receptor signalling. Nat Rev Immunol. 2004:4(7):499-511.

23. Ullah MO, Sweet MJ, Mansell A, Kellie S, Kobe B. TRIF-dependent TLR signaling, its functions in host defense and inflammation, and its potential as a therapeutic target. J Leukoc Biol. 2016;100(1):27-45.

24. Akira S. TLR signaling. Curr Top Microbiol Immunol. 2006;311:1-16.

25. Ramnath D, Powell EE, Scholz GM, Sweet MJ. The toll-like receptor 3 pathway in homeostasis, responses to injury and wound repair. Semin Cell Dev Biol. 2017:61:22-30.

26. Beutler B. Inferences, questions and possibilities in Toll-like receptor signalling. Nature. 2004;430(6996):257-63. 
27. O'Neill LA, Golenbock D, Bowie AG. The history of toll-like receptorsredefining innate immunity. Nat Rev Immunol. 2013;13(6):453-60.

28. Jiang Z, Mak TW, Sen G, Li X. Toll-like receptor 3-mediated activation of NF-kappaB and IRF3 diverges at Toll-IL-1 receptor domain-containing adapter inducing IFN-beta. Proc Nat Acad Sci USA. 2004;101(10):3533-38.

29. Lin Q, Wang L, Lin Y, Liu X, Ren X, Wen S, Du X, Lu T, Su SY, Yang X, et al. Toll-like receptor 3 ligand polyinosinic:polycytidylic acid promotes wound healing in human and murine skin. J Investig Dermatol. 2012;132(8):2085-92.

30. Subauste MC, Jacoby DB, Richards SM, Proud D. Infection of a human respiratory epithelial cell line with rhinovirus. Induction of cytokine release and modulation of susceptibility to infection by cytokine exposure. J Clin Investig. 1995;96(1):549-57
31. Zhu Z, Tang W, Gwaltney JM Jr, Wu Y, Elias JA. Rhinovirus stimulation of interleukin-8 in vivo and in vitro: role of NF-kappaB. Am J Physiol. 1997:273(4):L814-824

32. Lopez-Souza N, Favoreto S, Wong H, Ward T, Yagi S, Schnurr D, Finkbeiner WE, Dolganov GM, Widdicombe JH, Boushey HA, et al. In vitro susceptibility to rhinovirus infection is greater for bronchial than for nasal airway epithelial cells in human subjects. J Allergy Clin Immunol. 2009;123(6):1384-1390e1382.

\section{Publisher's Note}

Springer Nature remains neutral with regard to jurisdictional claims in published maps and institutional affiliations.
Ready to submit your research? Choose BMC and benefit from:

- fast, convenient online submission

- thorough peer review by experienced researchers in your field

- rapid publication on acceptance

- support for research data, including large and complex data types

- gold Open Access which fosters wider collaboration and increased citations

- maximum visibility for your research: over 100M website views per year

At BMC, research is always in progress.

Learn more biomedcentral.com/submissions 